\title{
Monocular stereopsis with and without head movement
}

\author{
HIROSHI ONO and MARTIN J. STEINBACH \\ York University, North York, Ontario, Canada
}

\begin{abstract}
Random dots moving with various velocity gradients were presented to observers; the motion was yoked to head movement in one condition and to no head movement in another. In Experiment 1, 12 observers were shown motion gradients with sine, triangle, sawtooth, and square waveforms with amplitudes (equivalent disparities) of $12^{\prime}$ and $1^{\circ} 53^{\prime}$. In Experiment 2, 48 observers were shown only the sinewave or square-wave gradient of $1^{\circ} 53^{\prime}$ disparity either with or without head movement so that the observers' expectation to see depth in one condition did not transfer to another. The main findings were: (1) with 12 ' disparity, the head-movement condition produced perceived depth but almost no perceived motion, whereas the no-head-movement condition produced both perceived depth and perceived motion; (2) with $1^{\circ} 53^{\prime}$ disparity, both conditions produced perceived depth and perceived motion; and (3) when the expectation to see depth was removed, the no-head-movement condition with the square-wave gradient produced no perceived depth, only motion. We suggest that monocular stereopsis with head movement can be achieved without perception of motion but monocular stereopsis without head movement requires perception of motion.
\end{abstract}

Monocular stereopsis can be demonstrated on a flat screen by a moving two-dimensional pattern yoked to lateral head movement or to lateral movement of the screen (Heine, 1905; Rogers \& Graham, 1979). It can also be demonstrated on a stationary screen by moving two-dimensional patterns shown to a stationary observer. The second kind of monocular stereopsis has been more extensively investigated and is known as the kinetic depth effect, stereokinetic effect, or motion perspective (see Braunstein, 1976; Kaufman, 1974; Regan, in press). In both cases, the impression of depth created is just as compelling as that created from binocular disparity cues. ${ }^{1}$

Using the random-dot technique pioneered by Rogers and Graham $(1979,1982)$, in this study we compared perceptions produced by motion on a two-dimensional screen when such motion was and was not yoked to head movement. To be more specific, the extent of perceived depth between the dots and that of perceived motion of the dots were compared. In the head-movement condition, horizontal movements of dots on a two-dimensional screen were yoked to lateral head movement. In the no-headmovement condition, the horizontal movements of dots were reproduced from the head-movement condition and thus the dots moved in the same way on the screen as in

This research was supported by Grants A0296 and A7664 from the Natural Sciences and Engineering Research Council of Canada, and by Grant EY05960 from the National Institutes of Health. The authors wish to thank T. Brooks for collecting the data and presenting them at the Association for Research in Vision and Ophthalmology, 1988. We also wish to thank G. Brock, E. Gonzalez, I. Howard, A. Mapp, M. Ohmi, B. Rogers, S. Saida, W. Simpson, M. Swanston, and N. Wade for their helpful comments on an earlier version of this paper. Correspondence may be addressed to Hiroshi Ono, Department of Psychology, York University, 4700 Keele St., North York, Ontario M3J 1P3, Canada. the head-movement condition. Such comparisons were recently made with a stimulus involving dynamic occlusion and motion parallax (H. Ono, Rogers, Ohmi, \& M. E. Ono, 1988); the present study involved a comparison of the perceptions of such stimuli without dynamic occlusion.

The two conditions are of interest for two reasons. First, the head-movement condition is known to produce perception of a stationary surface of dots when depth is seen. This stationary surface with depth can be thought of as being achieved with information about the head movement, namely (1) vestibular and proprioceptive signals, (2) the trapezoidal transformation of the screen, or (3) the retinal optic flow from the surrounding area of the screen. If any of the information is used to achieve this percept, there is no reason to expect the same percept when a similar extent of retinal motion is presented without these three pieces of information. Thus, in the no-head-movement condition, the perceived extent of dot motion was expected to be seen veridically. Second, it is not clear whether the stimuli used by Rogers and Graham (1979) with head (or screen) movement would lead to perception of depth when they are presented without head (or screen) movement. Their stimuli had a gradient of velocity within the screen that is known to produce depth (Braunstein \& Andersen, 1981; Braunstein \& Tittle, 1988; Flock, 1964; E. J. Gibson, J. J. Gibson, Smith, \& Flock, 1959), but they differed in detail from the depth stimuli used without head movement.

In Experiment 1, each observer served in the two conditions (head movement and no head movement) for four different kinds of stimulus movements (those used by Rogers \& Graham, 1979). In Experiment 2, different observers served in the two conditions so that the expectation derived from viewing the stimulus in one condition was not present in the other. 


\section{EXPERIMENT 1}

Ever since J. J. Gibson (1950) proposed that a gradient of velocity is important in slant perception, there have been numerous studies using different types of gradients. With head movement, four different gradients-sine, triangle, sawtooth, and square waveforms-were used by Rogers and Graham (1979). Without head movement, sawtooth and triangle waveforms have been used. In early experiments (e.g., Braunstein 1968; Flock, 1964), the stimulus elements were moved to simulate a slanted flat surface, which can be considered as one cycle of a sawtooth stimulus. In later experiments, (e.g., Braunstein \& Andersen, 1981; Farber \& McConkie, 1979), the stimulus elements were moved to simulate two flat surfaces meeting in front or in back, which can be considered as one cycle of a triangle stimulus. Although these two waveforms can be said to be the same as the two used with the head movement, there are differences in the stimulus presentations. In the previous experiments without head movement, the stimulus elements were moved in the same direction (excluding the elements that did not move), and the slower elements were usually perceived as being farther away than the faster ones. In the experiment with head movement, however, the different elements were moved in opposite directions. Elements that move in the same direction as the head are seen as being farther away, whereas those that move in the opposite direction are seen as being closer (M.E. Ono, Rivest, \& H. Ono, 1986; Rogers \& Graham, 1979, 1982).

In this experiment, both the head-movement and the nohead-movement conditions were presented with the four different velocity gradients used by Rogers and Graham (1979). Two different extents of relative motion for each gradient were presented. The large extent of relative motion was expected to produce perceived motion of the dots even in the head-movement condition, because beyond the range in which motion parallax is completely effective, motion is seen (M. E. Ono et al., 1986; Saida \& H. Ono, 1989). Observers were asked to report the extent of depth as well as that of the motion. In the present study, only the extent of depth was examined; for recent studies on the direction or sign of depth (i.e., what part of the stimulus appears closer), see Braunstein and Tittle (1988); H. Ono et al. (1988); and B. J. Rogers, Ono, and S. Rogers, (1988).

\section{Method}

Apparatus and Stimuli. An array of 512 random dots with a mean density of $3.9 \mathrm{dots} / \mathrm{cm}^{2}$ was presented on an oscilloscope (Tektronic Model 604) in a dimly lit room. Black cardboard was placed around the oscilloscope screen to reduce the cues about the twodimensionality of the display. The visible portion of the screen was $10 \times 13 \mathrm{~cm}$ and was viewed from a distance of $40 \mathrm{~cm}$. A chinrest constrained the observer's head to move horizontally along a track through a range of $15 \mathrm{~cm}$. Movement of the dots on the screen could be yoked to the movements of the chinrest. Head movement was also recorded for use on subsequent trials without head movement to move the dots. The relative dot motions within the displays had gradients of sine, triangle, sawtooth, or square waveforms. The number of cycles of the waveforms from the top to the bottom of the oscilloscope screen was set at two in all trials. The most rapidly moving dots moved 0.8 and $7.5 \mathrm{~mm}$ for the full head movement, and thus the two extents of relative dot motion were 0.16 (small) and $1.50 \mathrm{~cm}$ (large). The equivalent disparity (Rogers \& Graham, 1979) and simulated depth specified by the two relative motion conditions were $12^{\prime}$ disparity and $0.4 \mathrm{~cm}$, and $1^{\circ} 53^{\prime}$ disparity and $4.0 \mathrm{~cm}$, respectively.

Experimental design and conditions. For each observer, there were 64 trials: 2 (head movement and no head movement) $\times 2$ (12' disparity and $1^{\circ} 52^{\prime}$ disparity) $\times 4$ (waveform) $\times 4$ (trials). A block consisted of four waveforms presented randomly without replacement. For each waveform within the block, one of the two disparities was randomly chosen, with the restriction that by the end of the experiment, the disparities were equally represented, but headmovement and no-head-movement conditions were paired. Half of the pairings started with head movement, whereas the other half started with a no-head-movement condition.

Procedure. In each trial, the observer viewed the display monocularly with the preferred eye. A red dot at the center of the oscilloscope screen acted as a fixation point. Half of the trials were headmovement trials, in which the observer was asked to move his/her head continuously from one end of the chinrest track to the other end at a speed that felt comfortable. Thus, the extent of head movement, and therefore that of the relative dot motion, was the same for all observers. The other trials were no-head-movement trials, in which the dots moved while the observer remained stationary.

For a stimulus presentation of a given waveform and a given relative dot motion, the head-movement trial followed the no-headmovement trial or vice versa. The first trial of the experiment was a head-movement trial, but for all subsequent stimulus presentations, the order was randomly determined, with the restriction that the head-movement and no-head-movement trials were equally represented for each stimulus. When head-movement trial preceded the no-head-movement trial for a given stimulus condition, our method of recording the head movements allowed us to present identical relative dot motions on the screen for both conditions. When the no-head-movement condition came first, the recording of the head movement from the last trial with head movement was used to move the stimulus.

Each observer was asked to indicate (1) the amount of depth and (2) whether the dots appeared to translate. An observer who perceived motion was asked to choose dots at points that represented the "farthest" and "closest," and to report the distance that each dot appeared to move. For example, an observer who perceived motion while viewing the sinewave stimulus with head movement chose reference dots on the peaks and troughs and reported the perceived extent of motion of each dot. The observer indicated the perceived depth and motion by placing a thumb on a hand-held ruler which he/she visually checked before showing it to the experimenter. Trials lasted $60 \mathrm{sec}$ and the observers were told that a trial would be repeated at a later time if they were unable to complete it within the time limit. Two of the observers required that one trial be repeated.

Observers. Twelve observers from York University volunteered to participate. All were familiar with the apparatus and the procedure and had served in similar experiments.

\section{Results and Discussion}

There were two sets of analyses. The first examined the extent of perceived depth and the second examined the extent of perceived motion, for which the extents of perceived motion of the farthest dot and that of the closest dot were summed. Before both analyses were made, the means of perceived extents for both depth and motion for the head-movement trials preceding the no-head- 
movement trials in each subcondition were compared with those for the no-head-movement trials preceding the headmovement trials. Since there were no systematic differences, the data from these two types of trials were combined, and the mean of four responses for each subcondition was used as the basic unit of analysis. The group means are shown in Figures 1 and 2.

Extent of perceived depth. A three-way repeated measures analysis of variance was performed with (1) viewing condition (head movement and no head movement), (2) relative motion (12' and $1^{\circ} 53^{\prime}$ disparities), and (3) waveform (sine, triangle, sawtooth, and square) as factors. All of the interactions were statistically significant [viewing $\times$ relative motion $\times$ waveform, $F(3,33)=2.88$, $p<.05$; relative motion $\times$ waveform, $F(3,33)=12.39$, $p<.001$; viewing $\times$ relative motion, $F(1,11)=20.52$, $p<.001$; viewing $\times$ waveform, $F(3,33)=6.14$, $p<.05$ ]. All main effects were also significant [viewing, $F(1,11)=23.27, p<.001$; relative motion, $F(1,11)$ $=68.88, p<.001$; waveform, $F(3,33)=11.57$, $p<.001]$. These significant interactions and main effects together accounted for $70 \%$ of the variance.

Three of four interactions each accounted for less than $1 \%$ of the total variance. The three-way interaction is due to the two viewing conditions with the sinewave stimulus for the small relative-motion condition producing almost the same extent of perceived depth (see the two front bars on the far left in Figure 1 ). The viewing $\times$ relative motion interaction is due to the difference between the headmovement and no-head-movement conditions being larger in the large relative-motion condition (see Figure 3 ). The viewing $x$ waveform interaction is due to the differences between the head-movement and no-head-movement conditions being smallest with the sinewave stimulus. Note that the two smallest differences are in the far left group of bars in Figure 1. The relative motion $x$ waveform interaction accounted for $8 \%$ of the variance and will be discussed shortly.

The significant main effect of viewing - the primary interest of this study-accounted for $5 \%$ of the variance, and is due to the mean perceived depth in the headmovement condition being larger than that in the no-headmovement condition. This can be seen in Figure 1, in which the left bars of each pair are taller than the right bars. The obtained direction of difference is not easy to explain. For the head-movement condition, the exact expected depth $(0.4$ and $4.0 \mathrm{~cm})$ can be computed from the extents of relative motion on the retina, the extent of head movement, and the distance between the observer and the screen (M. E. Ono et al., 1986). In contrast, for the nohead-movement condition, it is not clear to us what assumptions should be made to compute the expected extent of depth. However, the fact that all of the no-headmovement conditions produced perceived depth indicates

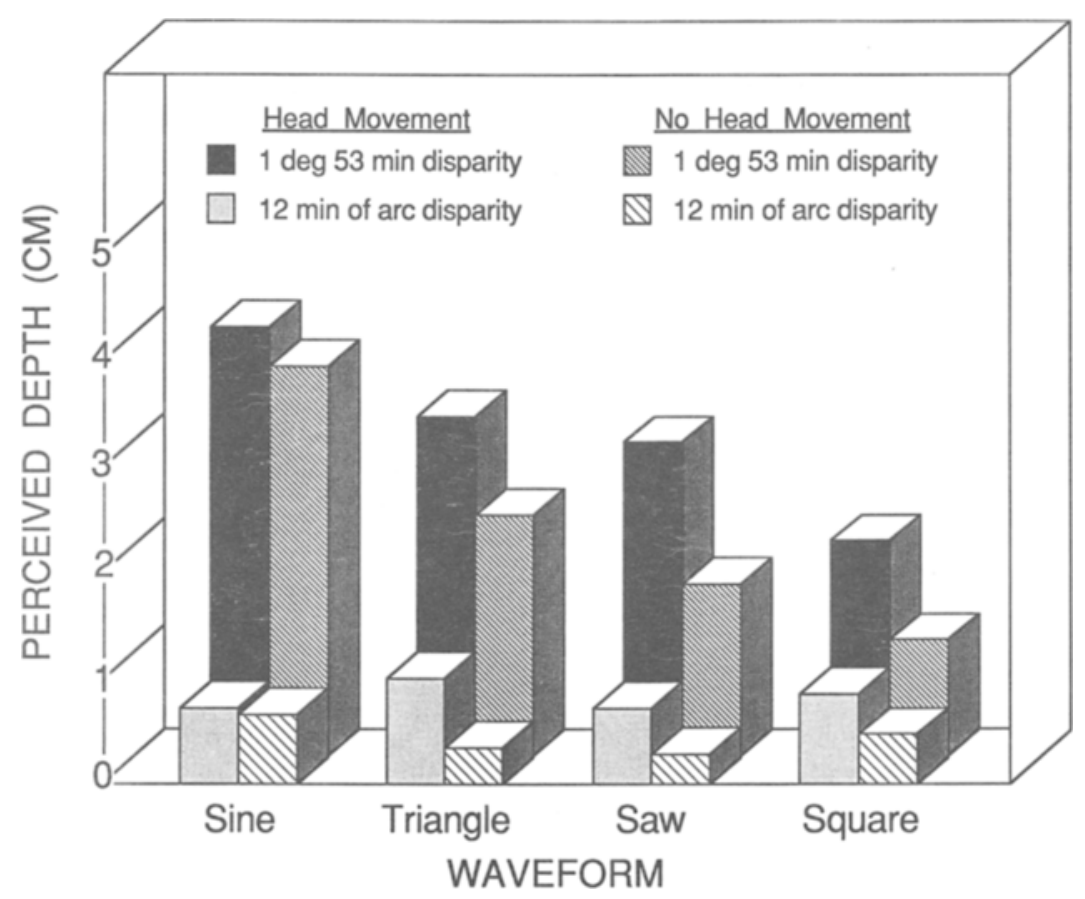

Figure 1. Extent of perceived depth as a function of head movement (left bars) versus no head movement (right bars), 6" disparity (front bars) versus 52" disparity (back bars), and profile of the gradient (sine, triangle, saw, or square waveform). Note that head movement always produced more apparent depth than did no head movement, that there was more perceived depth with the larger dot motion, and that there is a rank ordering of the amount of perceived depth with the waveform type, especially with the larger dot motion $(n=12)$. 


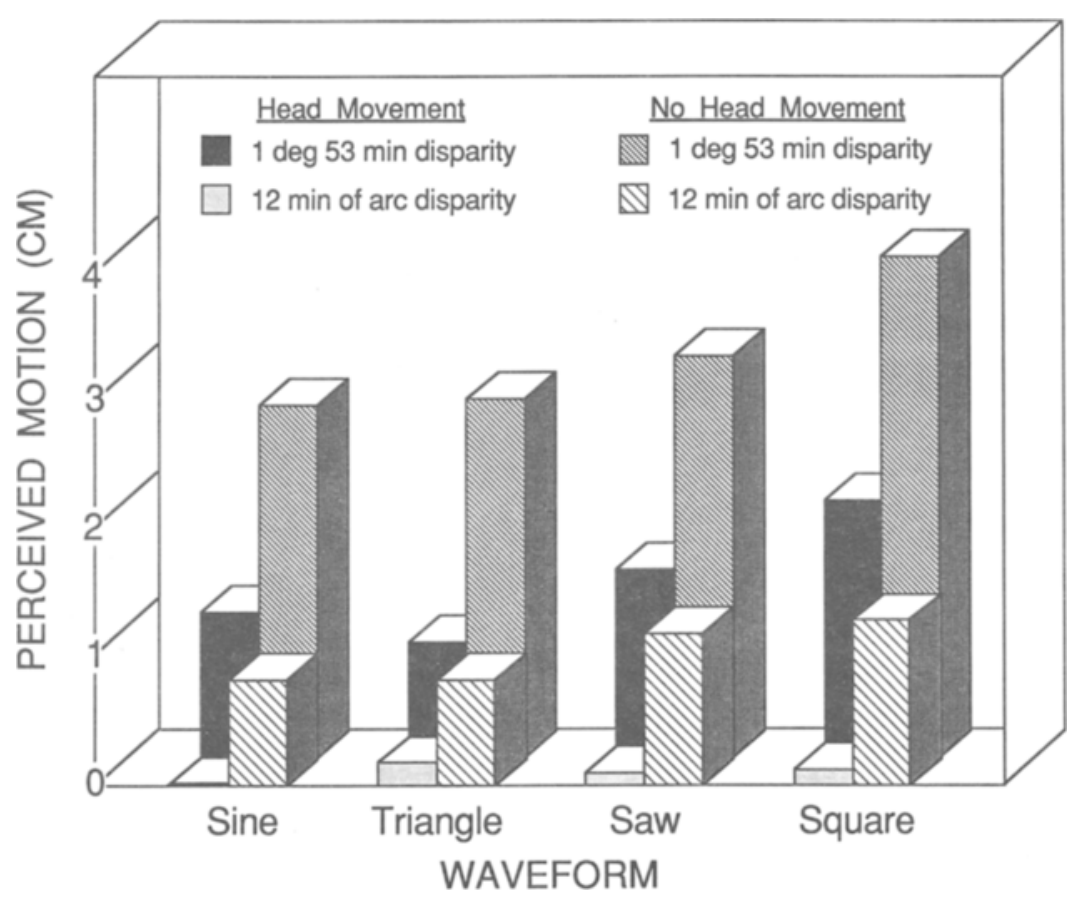

Figure 2. Extent of perceived motion as a function of head movement (left bars) versus no head movement (right bars), 6" disparity (front bars) versus 52" disparity (back bars), and profile of the gradient (sine, triangle, saw, or square waveform). Note that the amount of perceived motion is less in all of the head-movement conditions than in the no-headmovement conditions. The mean perceived motion is also less in the conditions in which the dot motion was smaller (and near zero when there was head movement). The waveform of the surface showed an effect on perceived motion that was the opposite of that found for the perceived depth (compare with Figure 1).

that the four stimuli used by Rogers and Graham (1979) can lead to depth perception without dot motion being yoked to head (or screen) movement.

The significant main effect of relative motion accounted for $47 \%$ of the total variance, and is due to the fact that the mean perceived depth in the small relative-motion condition was smaller than that in the large relative-motion condition. This effect can be seen in Figure 1, in which the front bars are shorter than the back bars in each pair of subconditions. The direction of this difference agrees with the difference in the simulated depth of the two relative-motion conditions with head movement. The results suggest that the greater the relative motion, the greater the depth. But this relationship has its limits. Recently, Saida and Ono (1989) found that the extent of perceived depth begins to decrease around $1^{\circ}$ of equivalent disparity with a square-wave stimulus when relative motion is yoked to head movement. Whether there is such a limit for relative motion not yoked to head movement remains uncertain.

The significant main effect of waveform accounted for 9\% of the variance, and is due to the larger mean perceived depth obtained in the following ranked order of waveforms: sine, triangle, sawtooth, and square. Figure 1 shows that this main effect is due primarily to the differences among the waveforms in the large relative-motion condition. Differences among the small relative-motion condition are very small. This lack of difference and the large differences among the waveforms in the large relative-motion condition accounted for the significant relative motion $x$ waveform interaction, which accounted for $8 \%$ of the variance.

One of the waveforms produced an unexpected result. The mean extent of perceived depth $(0.8 \mathrm{~cm})$ found in the no-head-movement condition with the square waveform was a surprise to us. Our expectation was that the observers would see no depth and would see different surfaces on the screen moving in opposite directions in a single plane, because the stimulus was essentially the same as that used in motion perception experiments. In fact, 8 out of 12 observers saw depth with small relative motion, and all 12 saw depth with large relative motion. The perceived depth in this condition could be due to an expectation to see depth. The head-movement and no-headmovement conditions were always paired for a particular stimulus presentation, and this procedure may have inadvertently provided the observers with an expectation to see depth in all of the no-head-movement conditions, 
particularly since we asked for the extent of depth in every trial. Experiment 2 was designed to minimize this effect of expectation.

Extent of perceived motion. A three-way repeated measures analysis of variance was applied to the extent of perceived motion using the same factors as in the analyses for the extent of perceived depth. Unlike the results of perceived depth, in which all the interactions were significant, only the relative motion $x$ waveform interaction was significant $[F(3,33)=4.61, p<.01]$. The nonsignificant interactions were viewing $\times$ relative motion $\times$ waveform $[F(3,33)=0.55]$ and viewing $\times$ waveform $[F(3,33)=0.69]$. However, as with the results for perceived depth, all of the main effects were statistically significant [viewing, $F(1,11)=36.41, p<.001$; relative motion, $F(1,11)=115.61, p<.001$; waveform, $F(3,33)$ $=4.61, p<.01]$. The significant interaction and the main effects together accounted for $62 \%$ of the variance. ${ }^{2}$

The significant interaction of relative motion $\times$ waveform accounted for only $1 \%$ of the variance, and is due to the differences between the small and large relativemotion conditions across waveforms. The largest difference between the small and large relative-motion conditions occurred with the square-wave stimulus $(1.11 \mathrm{~cm})$ and the smallest difference occurred with the trianglewave stimulus $(0.67 \mathrm{~cm})$. The theoretical significance of this interaction is not obvious to us.

The significant main effect of viewing - the primary interest of this study-accounted for $21 \%$ of the variance, and is due to the mean perceived motion in the headmovement condition being smaller than that in the nohead-movement condition. This difference can be seen in Figure 2, in which the left bar is shorter than the right bar in each subcondition (also see Figure 3). Note that there was almost no motion perception in the small relative-motion condition presented with a sinewave. In fact, 10 of the 12 observers reported no perceived motion in this condition. Furthermore, the same extent of screen motion led to a sizable extent of perceived motion in the no-head-movement condition.

The significant main effect of relative motion accounted for $33 \%$ of the variance, the mean perceived motion being smaller in the small relative-motion condition than in the large relative-motion condition. This is illustrated in Figure 2, in which the front bar is shorter than the back bar in each pair of subconditions (also see Figure 3). That a larger extent of motion on the screen led to a larger perceived motion is not surprising, but it is interesting to note that in the small relative-motion condition, the extent of perceived motion was near zero in the head-movement condition. This lack of perceived motion is consistent with the finding that no motion is seen in the range of equivalent disparities in which motion parallax is completely effective (H. Ono et al., 1988; M. E. Ono et al., 1986; Saida \& Ono, 1989).

The significant main effect of waveform accounted for $3 \%$ of the variance. The order of the size of mean perceived motion was opposite to that of perceived depth.
Greater extents of motion were perceived in the following ranked order of waveforms: square, sawtooth, triangle, and sine. This suggests a tradeoff of perceived motion and perceived depth across different stimuli. Such a tradeoff has been noted for the depth and motion seen with head movement (M. E. Ono et al., 1986), but why there should be such a tradeoff in the no-head-movement condition is not clear. (An analysis of the tradeoff across different subjects and across different experimental conditions is reported in Experiment 2.)

Our primary interest in this study was to examine whether or not motion perception was experienced, and we therefore did not ask the observers to report different types of motion. Experimenters serving as observers, however, noted that in the square-wave condition, the motion perception consisted of "shearing motion" (different surfaces moving in opposite directions), whereas in the other three conditions it usually consisted of "rocking motion" (a rigid three-dimensional corrugated surface rotating on a vertical axis). Thus, the "rigidity constraints" often invoked in computational analyses of vision to account for "structure from motion" were operating with three waveforms but not with the square-wave stimulus.

Our statistical analyses so far have been concerned with the differences in extents among conditions and have not directly examined the magnitudes themselves. An unexpected finding concerning the perceived motion was the overestimation in the no-head-movement conditions, in which veridical extents were expected. As described in the Method section, the most rapidly moving dots moved 0.8 and $7.5 \mathrm{~mm}$, and thus the extents of relative dot motion (or the sum of the extents of motion in opposite directions) were actually $0.16 \mathrm{~cm}$ in the $12^{\prime}$ disparity condition and $1.5 \mathrm{~cm}$ in the $1^{\circ} 53^{\prime}$ disparity condition. The means of the perceived extents however, were 1.0 and $3.1 \mathrm{~cm}$, respectively. These large discrepancies from the veridical magnitudes were confirmed with additional subjects, including the experimenters. We have no explanation for these discrepancies, but this exaggerated extent of perceived motion is not limited to this experiment. A small displacement in a random-dot kinematogram also produced an exaggerated extent (T. Sato, personal communication, November 1989).

Summary. The differences in the perceptions that occurred in the two viewing conditions are summarized in Figure 3. This figure shows the extents of perceived depth and motion averaged across the four waveforms. The head-movement condition produced greater perceived depth but smaller perceived motion than the no-headmovement condition. Note that the mean perceived motion in the small relative-motion condition is near zero.

These results show that, within limits, retinal motion produced by Rogers and Graham's technique, when coupled with head-movement information, leads to a perception of depth with no perception of motion. When an observer's head moves with respect to a simulated corrugated surface, the small associated retinal motion can 


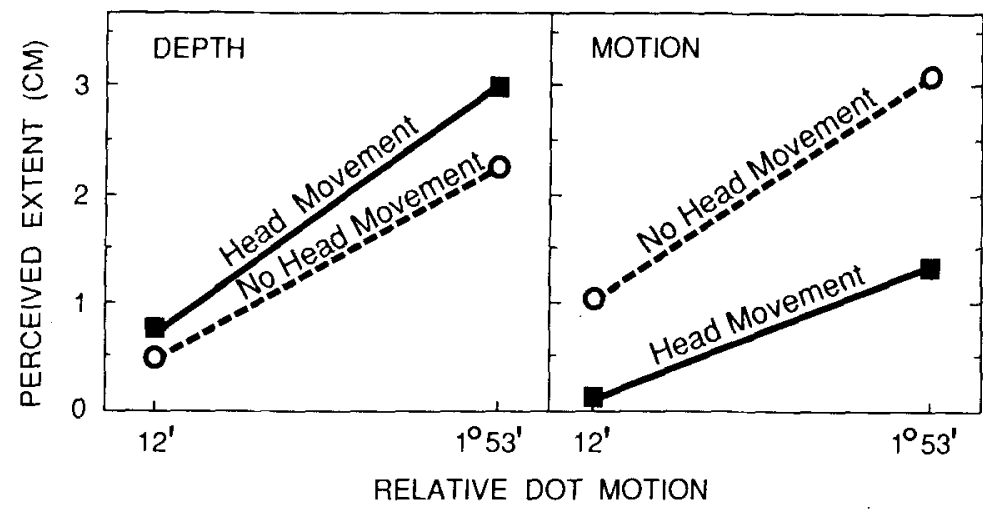

Figure 3. Summary of the results of Experiment 1 collapsed across the four waveforms. Perceived depth was greater with head movement, whereas perceived motion was greater with no head movement. Increasing the dot motion had the same effect of increasing both perceived depth and perceived motion.

produce a perception of depth with no motion. When the retinal motion is large, however, motion is seen, and location constancy is no longer experienced. Wallach (1985) called the area over which motion is not seen the "immobility range," and our data suggest that this range depends on the nature of the stimulus, since different waveforms produced different extents of motion.

The same stimulus on the screen presented without head movement led to unexpected motion and depth perception. The extent of perceived motion was larger than expected, and all four waveforms led to perceived depth. In particular, the perceived depth with the square-wave stimulus was a surprise. As mentioned before, this result may be due to the expectation to see depth resulting from the within-subjects design.

\section{EXPERIMENT 2}

To avoid transference of an expectation to see depth in one condition to another, a between-subjects design was used. We also recruited observers who had not previously participated in a depth-perception experiment.

\section{Method}

The same apparatus was used to present the stimuli as in Experiment 1 . However, of the four original waveforms, only the sine and square waveforms were used. The maximum dot motion was $7.5 \mathrm{~mm}$ for the full range of head movement, making the relative motion $1.5 \mathrm{~cm}$ or $1^{\circ} 53^{\prime}$ disparity, the same as in the large relativemotion condition of Experiment 1 . The procedure was the same as that used before, except that the observers in the no-headmovement condition were presented with the dot movements on the screen recorded from the last observer who was in the headmovement condition. Each observer was instructed on the procedure and given two practice trials. A 2 (head movement and no head movement) $\times 2$ (sine and square waveforms) factorial design was used, and each observer served in only one condition and was given four trials. Forty-eight students from York University volunteered to participate as observers. None of them had served in Experiment 1 or in any other depth-perception experiment.

\section{Results and Discussion}

The mean extents of depth and motion were computed for each observer the same way as in Experiment 1, and this served as the basic unit of analysis. The group means are shown in Figures 4 and 5.

A two-way analysis of variance was performed on the data for the extent of perceived depth with viewing condition (head movement and no head movement) and waveform (sine and square) as factors. Because there was no variance in one of the conditions, statistical significance levels are not reported here. The effect of the interaction accounted for less than $1 \%$ of the variance, and will not be discussed further. The two main effects accounted for $47 \%$ of the variance.

The effect of viewing accounted for $17 \%$ of the variance, and is due to the greater extent of perceived depth being seen with head movement. This effect can be seen in Figure 4, in which the left bars are taller than the right bars in the two waveforms. The right bar for the squarewave condition has a value of zero-none of the observers perceived depth in this condition. In contrast, in the same condition in Experiment 1, 10 out of 12 observers perceived depth. The strong difference in the no-headmovement conditions with square-wave stimulus in the two experiments suggests that the observers' expectation to see depth was responsible for the surprising finding in Experiment 1. When the expectation was minimized, no observer saw depth.

The fact that the no-head-movement condition with the square-wave stimulus produced perceived depth in Experiment 1 left open the possibility that the perceived depths seen in the three other no-head-movement conditions were also due to expectation. The perceived depth in the no-head-movement condition with the sinewave stimulus in this experiment, however, indicates that this is not the case, at least for the sinewave stimulus. That is, without any confounding expectation, depth was still seen with the sinewave stimulus in Experiment 2. 
The effect of waveform accounted for $30 \%$ of the variance, and is due to the greater perceived depth in the sinewave condition than in the square-wave condition. The direction of the difference of this main effect is in agreement with the results of Experiment 1. The sinewave stimulus was more effective for perception of depth than was the square-wave stimulus.

For the extents of perceived motion, a two-way analysis of variance was performed with viewing condition (head movement and no head movement) and waveform (sine and square) as factors. The interaction of viewing $\times$ waveform was not statistically significant $[F(1,44)=$ 0.21 , n.s.], but the two main effects were significant [viewing, $F(1,44)=9.91, p<.01$; waveform, $F(1,44)$ $=16.55, p<.001]$ and accounted for $34 \%$ of the variance. $^{3}$

The directions of difference in the two main effects were the same as those found in Experiment 1. The significant main effect of viewing accounted for $12 \%$ of the variance, and is due to the greater extent of motion being seen in the square-wave condition than in the sinewave condition. This effect can be seen in Figure 5, in which the right bars of each pair are taller than the left bars. The significant main effect of waveform accounted for $22 \%$ of the variance, and is due to the greater extent of motion being seen in the square-wave condition. This effect can be seen in Figure 5, in which the two right bars on the right side of the graph are taller than the two on the left side. These results confirm those of Experiment 1, in that the no-head-movement condition produced a greater extent of motion than did the head-movement condition and the square-wave condition produced a greater extent of perceived motion than did the sinewave condition.

The tradeoff of perceived depth and motion across stimulus conditions noted in Experiment 1 was also obtained in Experiment 2. That is, a greater extent of depth

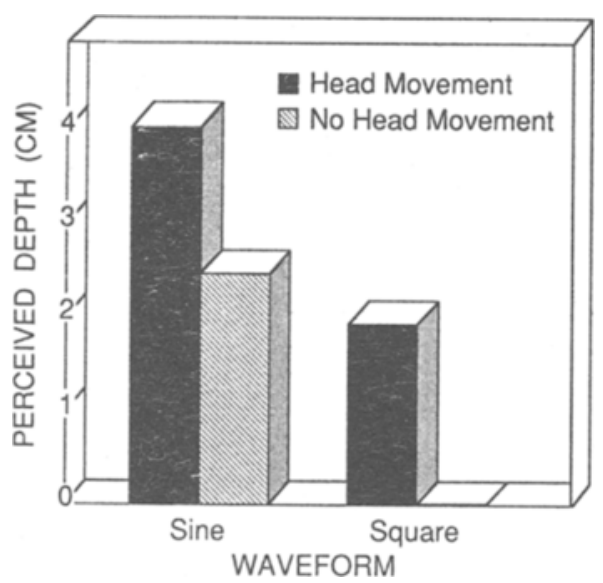

Figure 4. Perceived depth as a function of the source of retinal image motion (head movement vs. no head movement) and waveform (sine or square) in Experiment 2. As in Experiment 1, perceived depth was greater with head movement and with the sinewave stimulus. Independent observers were used in each of the four conditions ( $n=12$ per condition).

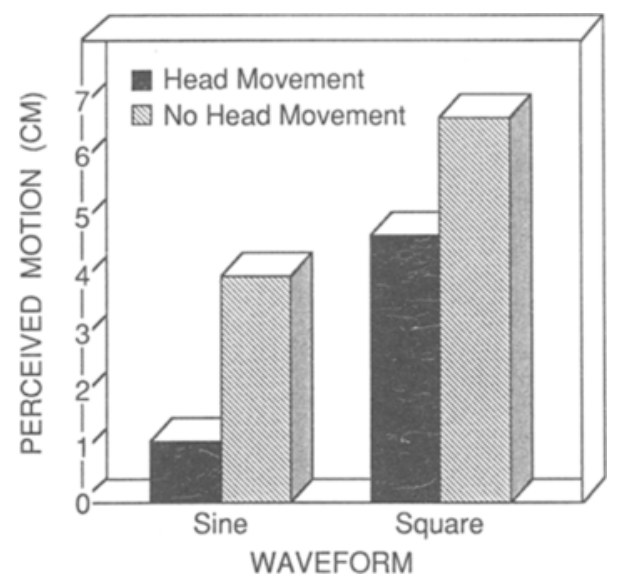

Figure 5. Perceived motion with and without head movement using the sinewave and square-wave stimuli. Greater motion is seen without head movement and in the square-wave condition, results similar to those found in Experiment 1 (compare with Figure 2).

was perceived with the sinewave stimulus than with the square-wave stimulus, but a greater extent of motion was perceived with the square-wave stimulus than with the sinewave stimulus. Moreover, there were within-condition tradeoffs across different observers as well. In Experiment 2 , the head-movement condition produced statistically significant negative correlations between perceived depth and perceived motion [square-wave stimulus, $r(11)$ $=-0.79, p<.005$; sinewave stimulus, $r(11)=-0.59$, $p<.025$ ]. In Experiment 1, eight different subconditions with head movement produced six negative and two positive correlations $(d f=11)$, ranging from -0.64 to +0.10 . These correlations suggest that for a given relative retinal motion yoked to head movement, what is not seen as depth is seen as motion. Although not easy to explain, there were similar trends in some of the no-headmovement conditions. There were fewer negative correlations, but some of them were statistically significant. In Experiment 2, the no-head-movement condition with the square-wave stimulus produced $r=0$ (no variance in perceived depth) and with the sinewave stimulus, $r(11)$ $=-0.61, p<.025$. In Experiment 1 , eight different subconditions without head movement produced three negative and five positive correlations, ranging from -0.53 to +0.56 .

More germane to the aim of Experiment 2, the results of perceived depth and motion from this experiment, taken together, explain the depth seen in the no-head-movement condition with the square-wave stimulus in Experiment 1. The observers' expectation to see depth in Experiment 1 was responsible for this result. When the expectation was removed, motion was seen exclusively. The retinal motion in this condition can come from different surfaces moving in opposite directions at different distances. In fact, there are infinite combinations of relative distances and velocities that can produce this retinal motion; that is, the retinal motion is ambiguous. When there is an ex- 
pectation to see depth, the visual system somehow chooses one of the possible combinations. When there is no such expectation, it chooses another possibility-namely, motion and no depth.

\section{GENERAL DISCUSSION}

The present results show clear differences in perception from the same stimulus movement on the screen depending on whether or not it was produced by an observer's head movement. A greater extent of depth was perceived with head movement than with no head movement; a greater mean extent of motion was perceived with no head movement than with head movement. The extents of perceived depth and motion also depended on the gradients of velocity. A greater extent of perceived depth was seen in the order of sine, triangle, sawtooth, and square waveforms, and that of motion was in the opposite order. The effects of different waveforms on the extents of depth and motion were not the same with and without head movement, however. The sine waveform was just as effective in producing depth with and without head movement when the retinal motion was small, but the extent of perceived motion was almost zero with head movement, whereas a relatively large extent of motion was seen without head movement. When the expectation to see depth was removed, the square waveform was effective for depth only when viewed with head movement; without head movement, no depth was seen, only motion.

The fact that some head-movement conditions did not produce motion perception, but all the no-head-movement conditions did, suggests that different mechanisms are involved in monocular stereopsis with and without head movement. In a traditional view, seeing depth without perceived motion represents location constancy (see Graham, 1951; Ittelson, 1960; Mack, 1986; Swanston \&Wade, 1987; Wallach, 1985), and the retinal motion is thought to be "compensated for" by information about the head movement. Presumably, the afferent signals from the retinal motion in the head-movement and no-head-movement conditions are coded by the same motion detectors, but at some stage the visual system must convert the motion signal to create a perception of a stationary surface when the retinal motion is produced by head movement.

A more recent interpretation is that head movement versus no head movement represents "conditions of common motion relative to the observer and no common motion relative to the observer" (one of the reviewers of this paper). That is, head movement can be thought of as adding a velocity vector to the stimulus on the screen relative to the head position (Braunstein \& Tittle, 1988) and as providing independent information about the changing position of the head relative to the screen. In the headmovement conditions, there were retinal optic flows from the experimental room as the head moved from side to side. Covarying with this optic flow is the trapezoidal transformation of the screen, and this transformation can also be important (Koenderink, 1985; Rogers \& Collett,
1989; Rogers \& Koenderink, 1986). The question as to whether the common motion or the transformation produces "direct" perception of stationary surfaces is avoided here, but these two variables can be considered to be providing information about head movement for location constancy.

Considering them as information places the traditional and the recent view on the same footing, and allows for testing whether they are in fact involved when the head (or the screen) moves. Such tests were recently conducted by Rogers et al. (1988), who presented the motion of random dots yoked to (1) head movement and the movement of the visual field pivoting on a vertical axis in the middle of the screen, (2) the rotation of the screen to produce the retinal transformation of the screen while the head was stationary, (3) the movement of the visual field as in (1) while the head was stationary, and (4) movement of the visual field pivoting on the vertical axis of one eye. They found that all four conditions disambiguated the direction of depth, whereas the same motion on a stationary screen with the head being stationary did not. Thus, they concluded that the vestibular signal, the trapezoidal transformation, and the optic flow from the surrounding area created by head or screen movements can be used by the visual system. Their study suggests that these pieces of information can be used to produce location constancy.

The idea that the motion signal is converted into depth perception with head-movement information is consistent with our results showing less motion with head movement. It is also consistent with the results showing depth/motion tradeoffs across conditions and across subjects in the head-movement conditions. These results agree with recent findings that the threshold of perceived motion is higher than the threshold of perceived depth when the motion on the screen is yoked to head movement (Saida \& Ono, 1989; Steinbach, Ono, \& Wolf, in press). When the retinal motion signal is completely converted into depth, monocular stereopsis with head movement occurs without a perception of motion.

As the terms "kinetic depth" and "structure from motion" imply, a necessary condition for monocular stereopsis without head movement is a perception of motion (for an explicit assumption of this, see Mather, 1989). In the case of monocular stereopsis with the movement of the screen or a "window" with dots moving at equal velocities in opposite directions (Braunstein \& Tittle, 1988; Rogers \& Graham, 1979), it is probably necessary for the screen or window to appear to move in order to see the depth from the motion within the screen. In contrast, monocular stereopsis with head movement can be achieved without a perception of motion, as the results of this study clearly indicate.

\section{REFERENCES}

Braunstein, M. L. (1968). Motion and texture as sources of slant information. Journal of Experimental Psychology, 78, 247-253.

Braunstein, M. L. (1976). Depth perception through motion. New York: Academic Press. 
Braunstein, M. L., \& Andersen, G. J. (1981). Velocity gradients and relative depth perception. Perception \& Psychophysics, 29, $145-155$.

Braunstein, M. L., \& Tittle, J. S. (1988). The observer-relative velocity field as the basis for effective motion parallax. Joumal of Experimental Psychology: Human Perception \& Performance, 14, $582-590$.

Cutting, J. E. (1986). Perception with an eye for motion. Cambridge, MA: MIT Press.

FARBER, J. M., \& McConkiE, A. B. (1979). Optical motions as information for unsigned depth. Joumal of Experimental Psychology: Human Perception \& Performance, 5, 494-500.

FLoCk, H. R. (1964). Some conditions sufficient for accurate monocular perceptions of moving surface slants. Joumal of Experimental Psychology, 67, 560-572.

Gibson, E. J., Gibson, J. J., Smith, O. W., \& Flock, H. (1959). Motion parallax as a determinant of perceived depth. Journal of Experimental Psychology, 58, 40-51.

GiBson, J. J. (1950). The perception of the visual world. Boston: Houghton Mifflin.

Graham, C. H. (1951). Visual perception. In S. S. Stevens (Ed.), Handbook of experimental psychology (pp. 868-920). New York: Wiley.

HEINE, L. (1905). On perception and conception of distance differences. Albrecht von Graefes Archiv für Klinische und Experimentelle Opthamologie, 61, 484-498.

ITTELSON, W. H. (1960). Visual space perception. New York: Springer.

Kaufman, L. (1974). Sight and mind: An introduction to visual perception. New York: Oxford University Press.

Koenderink, J. J. (1985). Space, form and optical deformations. In D. J. Ingle, M. Jeannerod, \& D. N. Lee (Eds.), Brain mechanisms and spatial vision (pp. 31-58). Dordrecht: Martinus Nijhoff.

LEE, D. N. (1974). Visual information about locomotion. In R. B MacLeod \& H. L. Pick, Jr. (Eds.), Perception: Essays in honor of James J. Gibson (pp. 250-267). Ithaca, NY: Cornell University Press.

MACK, A. (1986). Perceptual aspects of motion in the frontal plane. In R. K. Boff, L. Kaufman, \& J. P. Thomas (Eds.), Handbook of perception and human performance (pp. 17-1-17-38). New York: Wiley.

MATHER, G. (1989). Early motion processes and the kinetic depth effect. Quarterly Journal of Experimental Psychology, 41A, 183-198.

Ono, H., Rogers, B. J., Ohmi, M., \& ONo, M. E. (1988). Dynamic occlusion and motion parallax in depth perception. Perception, 17, 255-266.

ONo, M. E., RIvest, J., \& ONO, H. (1986). Depth perception as a function of motion parallax and absolute-distance information. Journal of Experimental Psychology, 12, 331-337.

REGAN, D. M. (in press). Depth from motion and motion in depth. In D. Regan (Ed.), Vision and visual dysfunction: Vol. 10A. Binocular vision. London: Macmillan.

ROGERS, B. J., \& COLLETT, T. S. (1989). The appearance of surfaces specified by motion parallax and binocular disparity. Quarterly Journal of Experimental Psychology, 41, 697-717.
Rogers, B. J., \& Graham, M. (1979). Motion parallax as an independent cue for depth perception. Perception, 8, 125-134.

Rogers, B., \& Graham, M. (1982). Similarities between motion parallax and stereopsis in human depth perception. Vision Research, 22, 261-270.

Rogers, B. J., Koenderink, J. (1986). Monocular aniseikonia: A motion parallax analogue of the disparity-induced effect. Nature, 322, 62-63.

Rogers, B. J., ONo, H., \& Rogers, S. (1988). The role of visual and non-visual information in disambiguating motion parallax transformations. Investigative Ophthalmology \& Visual Science, 29(Suppl. 3), 265.

SAIDA, S., ONO, H. (1989). What extent of motion parallax produces depth perception? Abstract submitted for presentation.

SedGwick, H. A. (1986). Space perception. In R. K. Boff, L. Kaufman, \& J. P. Thomas (Eds.), Handbook of perception and human performance (pp. 21-1-21-57). New York: Wiley.

Steinbach, M. J., ONo, H., \& Wolf, M. E. (in press). Motion parallax judgements of depth as a function of the direction and type of head movement. Canadian Journal of Psychology.

Swanston, M. T., \& WADE, N. J. (1987). The perception of visual motion during movements of the eyes and of the head. Perception \& Psychophysics, 43, 559-566.

Swanston, M. T., Wade, N, J., \& Day, R. D. (1987). The representation of uniform motion in vision. Perception, 16, 143-159.

Wallach, H. (1985). Perceiving a stable environment. Scientific American, 252, 118-124.

\section{NOTES}

1. Discussions in this paper are limited to perception related to a lateral movement of the head and of the stimulus. For discussions of more general cases, see Braunstein (1976), Mack (1986), Sedgwick (1986), and Swanston, Wade, and Day (1987). For discussions of retinal motion providing information for movement of the body, see Cutting (1986) and Lee (1974).

2. A four-way analysis of variance, which included the factor plane (near and far), was also performed. The extents of perceived motion of the "farthest" and "closest" points were statistically different, and this factor interacted with others [plane, $F(1,11)=10.30, p<.01$; viewing $\times$ plane, $F(1,11)=13.45, p<.01$; relative motion $\times$ plane, $F(1,11)=8.76, p<.01$; viewing $\times$ relative motion $\times$ plane, $F(1,11)$ $=11.90, p<.01]$. As we shall see, these statistical significances may have theoretical importance, if "shearing" and "rocking" motions are associated with different subconditions.

3. Unlike in Experiment 1, the main effect of plane was not statistically significant, $[F(1,44)=0.05]$, and this factor did not interact with the other two factors [viewing $\times$ plane, $F(1,44)=0.25$; waveform $\times$ plane, $F(1,44)=1.89$; viewing $\times$ waveform $\times$ plane, $F(1,44)=1.30$ ].

(Manuscript received February 16, 1989: revision accepted for publication February 13, 1990.) 